\title{
The Long and the Short of It: Maximizing the Impact of Public Service Announcements in the Age of the "Twitterverse"
}

\author{
Harper Roehm Jr. ${ }^{1}$ \\ ${ }^{1}$ Marketing, Entrepreneurship, Hospitality and Tourism, Bryan School of Business and Economics, University \\ North Carolina at Greensboro, Greensboro, NC, United States \\ Email: haroehm@uncg.edu
}

\begin{abstract}
This article reports the results of an empirical study of current public service announcements. We apply a framework of communication norms espoused by Grice (1975) to identify a structural dimension of public service announcements - conciseness - that has been relatively understudied and that may be crucial to understanding the level of audience impact that is achieved. Further, recognizing the evolving concerns of sponsors in a marketplace flooded with novel modes of communication, we examine potential differences in the dynamics of conciseness as a function of the conventions of "new" (e.g., web-based) versus "traditional" (e.g., standard television, print) media environments. Results garnered from testing two forms of new media and two forms of traditional media suggest that persuasiveness varies with message length in opposing directions. For new media, messages that were briefer created a stronger impression, whereas for messages in traditional media, greater duration corresponded to greater impact.
\end{abstract}

Keywords: Public Service Announcements; new media; communication norms.

\section{Introduction}

Few would argue against such ethical and socially responsible activities as aiding disaster relief, supporting medical research, mentoring at-risk children, conserving natural resources, wearing seatbelts, stopping a smoking habit or preventing someone from driving under the influence of alcohol. Still, human beings are fallible, and as such, the reality of behavior often lags behind ideals. We waste water by delaying the repair of a leaky faucet. We neglect to buckle up. We bite our tongues when colleagues insist they can drive after imbibing.

Public service announcements are a means by which the business community helps to address gaps between ethical and socially beneficent goals and individuals' behaviors in practice. Often created through the pro bono efforts of professional marketers and advertisers, public service announcements provide business organizations with a powerful mechanism for educating the populace and mobilizing positive action. In doing so, public service announcements also serve to engage and frame public conversations around issues of social and ethical relevance. Consider, for example, the iconic "Friends Don't Let Friends Drive Drunk" campaign. On one hand, it has armed citizens to manage the personal ethical dilemma of whether and how to intervene when someone intends to drive while impaired. At the same time, at an aggregate level, it has also raised the collective consciousness of our joint obligation to protect fellow drivers and keep the roads safe for all users.

Although the attributes and effects of public service announcements have received significant scholarly attention, many questions remain regarding their efficacy (Fishbein et al. 2002; Murry et al. 1996). Moreover, the emergence in recent years of novel media alternatives has ushered in new challenges and opportunities that, as yet, remain largely unexplored by academic researchers in this domain (Walther et al. 2010). This article reports the results of an empirical study of current public service campaigns that is intended to expand our grasp of this important bridge between the business sector and the wellbeing of our culture and society.

Applying a prominent theory of communication norms as a lens, our analysis will identify a structural dimension of public service announcements - conciseness - that has been relatively understudied and that may be crucial to understanding the level of audience impact that is achieved. Further, recognizing the challenges faced by sponsors of public service announcements in a marketplace flooded with new 
modes of communication, we will examine potential differences in the dynamics of conciseness as a function of the conventions of "new" (e.g., web-based) versus "traditional" (e.g., standard television, magazine) media environments. After deriving and testing two primary hypotheses, we will discuss the contribution of the findings to both the scholarship and practice of managing these good works.

\section{Background}

\subsection{Public Service Announcements: A Brief History}

Public service announcements are noncommercial messages aimed at improving knowledge, attitudes and/or behavior on socially desirable dimensions (Martiniuk et al. 2010; Murry et al. 1996). In the United States, the modern era of public service announcements dates back to World War II, although advertisements addressing national and social issues began years before. For example, newspaper advertisements recruited soldiers and solicited funds during the Civil War.

In November 1941, a writer and university professor named James Webb Young articulated an idea for advertising that would serve the public interest. He envisioned its application as a powerful tool for helping people, and at the same time, rebuilding respect for American business in the aftermath of the Great Depression.

"It ought to be used for open propaganda in international relations, to create understanding and reduce friction. It ought to be used to wipe out such diseases of ignorance as childbed fever. It ought to do the nutritional job this country needs to have done. It ought to be the servant of music, of art, of literature and of all the forces of righteousness..." (Young 1941, cited the Ad Council's 2002 report, "Matters of Choice: Advertising in the Public Interest," p. 3).

Young's insight led to the development of a model in which public service announcements would be sponsored by businesses, which would "loan" the time and talent of communication professionals to develop and execute the messages. This is an approach that continues to thrive in the U.S. today. Businesses also provide other critical forms of support for public service announcements, including free media space to carry the messages and philanthropic donation of funds to cover campaign operating expenses. The fruits of these labors - ranging from admonitions in the 1940s that "Loose Lips Sink Ships" to later entreaties to "Keep America Beautiful," support the Peace Corps, prevent the spread of AIDS, and thwart domestic violence - have been recognized as some of the most visible demonstrations of corporate willingness to assume social responsibility.

In some cases, public service campaigns have been remarkably successful. For example, per statistics provided by the Ad Council (a non-profit organization that organizes public service announcement efforts in the U.S), after the launch of a long-running effort to educate the populace on the benefits of safety belts, usage grew from $14 \%$ to $79 \%$, saving an estimated $\$ 3.2$ billion in costs to society. In response to an "environmental defense" campaign, the amount of waste recycled increased $385.4 \%$ from the 1980s through the turn of the century. Communications on behalf of Big Brothers Big Sisters have caused mentor applications to soar from 90,000 per year to 620,000. Furthermore, an impressive $68 \%$ of Americans say that they have personally stopped someone from driving after s/he had been drinking.

Nonetheless, the effects of public service announcements have not been universally positive. Scholarly investigations suggest that results can be quite variable, and in fact, that ill-conceived campaigns may even produce a "boomerang effect" that undermines inclinations to change a behavior in the socially desirable direction (e.g., Fishbein et al. 2002; O'Dea 2005). It is critical, therefore, to identify factors that may moderate the efficacy of public service announcements, so that the intended impacts on target audiences can be achieved.

Significant progress has been made in this regard. Research has revealed, for instance, that the effects of public service announcements are enhanced by the following elements: alignment of tone and style to target audience mindset, so that for example, consumers with a high need for stimulation receive messages that have an exciting and novel look at feel (Lorch et al. 1994; Morgan et al. 2003; Zhao and Pechmann 2007); vivid demonstrations, rather than mere descriptions, of aspirational behaviors (Martiniuk et al. 2010; Morgan et al. 2003); employment of credible protagonists, who, say, are actual sufferers of focal diseases (Shanahan et al. 2010; Siu 2010; Toncar et al. 2007); active engagement of 
negative stereotypes and feelings of disgust for those who violate prescribed actions, such as smokers (Pechmann and Knight 2002; Pechmann and Reibling 2006).

However, even as such research begins to disambiguate the elements of success for public service announcements, it must be admitted that the ability to speak to issues faced by today's message framers is somewhat limited by a common denominator among many extant studies. This is, namely, a focus on messages placed in what we shall term "traditional" media sources. By this, we refer to such mature and conventional vehicles as television, magazines, and newspapers. By contrast, examination of public service announcements within the context of "new" media - by which we refer to such novel and emergent alternatives as web-facilitated and mobile media - has been rare (for an exception, see Walther et al. 2010). The present research contributes to the closure of this fissure in our knowledge base.

\subsection{Theoretical Framework and Prior Research}

As foundation for examining the effectiveness of public service announcement messages in new media, it will be useful to consider what sorts of factors may influence the manner in which a public service announcement is received by an audience member. Guidance on this matter may be derived from a wellregarded treatise by Grice (1975) on the pragmatics of communication. Grice enumerates several maxims that govern the way in which human beings interpret messages to which they are exposed. Specifically, it is expected at a minimum that the substance of a communiqué will adhere to the following principles: It will be relevant to our needs, be truthful, be clear and be as concise as is feasible. When any of these expectations is violated, the persuasive effect of a message, such as a public service announcement, may be undermined.

Prior research on public service announcements can be interpreted as consistent with the first three of Grice's maxims. For example, bearing out the principle of relevance, anti-smoking research indicates that intentions to not smoke in the future are most impacted by advocacy messages that are germane to the motivational sensibility of the target audience, and in particular, whether their goals are to aspire to positive outcomes, such as good health, or to prevent negative outcomes, such as disease (Zhao and Pechmann 2007). Veracity is also critical, as illustrated by research that documents stronger attitudes toward a public service announcement, and greater intentions to donate to its focal causes, when truth value is underscored by featuring actual victims of disadvantageous actions (smoking-related cancer, death from drunk driving) rather than actors (Shanahan et al. 2010). Finally, the significance of clarity is exemplified by research showing that intentions and reported pro-social behaviors are enhanced by messages that are vivid and explicit about consequences of noncompliance (e.g., health complications and negative social consequences from smoking) and provide a concrete means of response such as a tollfree number to call (Anand Keller and Block 1999; Pechmann and Reibling 2006; Roberto et al. 2002).

At the same time, evidence related to the fourth Gricean dictate--that is, on how conciseness may affect the ability of a public service announcement to influence ethical and socially responsible behavior-is less readily available and thus is the focus of our investigation. In examining this axiom, we begin with the observation that it may possess a logical difference from the other three, in that what is viewed as satisfactory may be less naturally predefined. To wit, while people may customarily accept nothing less than high levels of relevance, clarity and truth in persuasive communications, what constitutes an appropriate degree of conciseness, or message length, may be less obvious at the surface. In support of this proposition, it may be noted that a substantial body of psychological research has demonstrated that individuals vary both interpersonally and situationally in their preferences for the amount of information or detail (Cacioppo and Petty 1982; Chaiken and Eagly 1983; Chaiken et al. 1989; Petty and Cacioppo 1986a, 1986b; Petty et al. 1983; for a review, see Eagly and Chaiken 1993, Chapter 7).

For members of the marketing community who may be involved with planning and shaping a public service announcement, the implication is therefore that there may be no simple rule of thumb for calibrating the proper level of message conciseness. Rather, the challenge may be to identify what sorts of factors lead audience members to respond favorably to a briefer or more expansive public service announcement and then to size a communiqué accordingly.

We propose that a major influence in this regard will be the media environment in which a message is delivered. As one flips through a mental archive of memorable public service announcements - Smokey Bear warning that "Only You Can Prevent Forest Fires," the Crash Test Dummies demonstrating that "You Could Learn A lot from a Dummy," the wake-up call inherent in "A Mind Is a Terrible Thing to 
Waste" - the powerful use of "traditional media" (television commercials, print advertisements) is apparent. Increasingly, though, ethical and socially responsible advocacies are being placed in "new" media (Walther et al. 2010), such as the as web-mediated and mobile alternatives that now are at the consumer's disposal. Along these lines, recent campaigns for childhood adoption, avoidance of home foreclosures, wildfire prevention, help for teen dating violence and many other causes have begun to include online videos, web banners, social network pages, and the like.

\subsection{Media Context and Public Service Announcements}

Successful use of new media options to deliver public service announcements requires an understanding of, and respect for, the conventions of the contexts. To appreciate and understand the nature of these norms, it may be helpful to consider the values that are projected by major institutions that structure the domain. Along these lines, one of the most profound influences on the new media landscape is Twitter, a social networking and microblogging service that enables its users to send and read very short text-based messages. With a tight 140-character constraint on missive size, Twitter explicitly endorses a succinct, pithy approach to communication that, given its current cultural prominence, may be argued to shape more general expectations about the appropriate parameters of messaging in digital and mobile media.

The broader effects of this philosophy on the way information is packaged and shared have been bemoaned as the "Twitterization" of communication (e.g., Hayes 2009), a term that has been coined to signify that people are becoming more and more accustomed to reducing information consumption to small, bit-sized chunks that can be easily transmitted to others. Moreover, as an indicator of the degree to which an assumption of efficiency has become embedded in our use of new media, consider the evolving shorthand of personal expression that pervades formats such as social media and texting. Examples would include the expanding application of "initialisms," in which beginning letters are combined to represent entire words (such as "LOL" for "laugh out loud"), the frequent omission of nonessential letters such as vowels ("lv" for "love," for instance), and the uses of irregular spellings with homophones (for example, "b4" for "before" or "gr8" for "great"), and so forth (Jones and Schieffelin 2009).

Taken as a whole, this discussion suggests that there may be an inherent preference for, and supposition of, conciseness regarding communications that appear in new media. It follows that organizations that seek to optimize the impact of a public service announcement on ethical and socially responsible behaviors may be well-advised to adhere to a criterion of brevity in creating messages for such channels. In such contexts, less may often be more.

Stated more explicitly, the suggestion is that messages housed in new (e.g., web-oriented or mobile) media should be better-received, and thus should more positively affect feelings about engaging in ethical and socially responsible behaviors, when they are more terse in length. This prediction is summarized in the following hypothesis:

H1: After exposure to public service announcements appearing in new media, audience attitudes will be more favorable when message length is relatively short, but less favorable when message length is relatively long.

By contrast, given audience members' long prior histories and ingrained, pre-existing habits of consumption of traditional (e.g., television, magazines, newspapers) media, the new-media preoccupation with conciseness may not carry over. Instead, for public service announcements placed in traditional media, a perhaps more intuitive wisdom of "more is better" may be expected to apply. That is, absent the expectation of brevity that may dominate new media, and provided that audience members are willing to devote sufficient time and attention, a lengthier public service announcement placed in a traditional medium may be more persuasive. This is because a longer message may be better able to convey more information about the ethically and socially desirable behavior that is being advocated. Accordingly, in traditional media, a longer message may produce stronger attitudes toward the recommended course of action. Our second hypothesis captures this logic.

H2: After exposure to public service announcements appearing in traditional media, audience attitudes will be more favorable when message length is relatively long and less favorable when message length is relatively short.

Our two hypotheses were tested in an empirical study, which is reported next. 


\section{Study}

To test our predictions, we measured consumers' responses to executions from current campaigns sponsored by the Ad Council. For the purposes of the study, exposure occurred in a controlled lab setting, in order to minimize extraneous variance that might arise from viewing distractions.

\subsection{Respondent Sample}

One hundred fifty-two adult volunteers were recruited via posters inviting involvement in a study of public service announcements. In appreciation for their time, all respondents received a gift card for a free beverage at a coffee house (approximate value $\$ 5$ ).

\subsection{Public Service Announcement Selection}

In order to enhance the generalizability of our findings, it was desirable to include multiple examples of specific messages and advocated causes. Public service announcements included in the study were sampled from the current archives of the Ad Council. At the time of the study, 50 ongoing campaigns were available for public examination. In determining the final set of messages to be tested, five criteria were applied. The campaign must:

1)include primarily English language executions (messages in other languages were omitted from testing);

2)relate to a broad adult population; this criterion eliminated campaigns aimed solely at certain subgroups, such as children, residents of specific geographic areas (e.g., New York City, the Gulf of Mexico region), veterans of the armed services and actual sufferers of specific diseases (e.g., arthritis)

3)incorporate, at a minimum, messages presented in two traditional (television, print) and two new (web banners, online videos) media

4)offer online videos that featured original content and were not simply a reposting of the television commercials; for example, a financial literacy campaign that was involved in the study provided how-to videos on issues such as "fraud prevention" and "choosing the correct bank for you"

5)make use of messages of different lengths, for example, featuring television commercials that varied from 15 to 30 seconds in length

Two campaigns, encompassing a total of 24 separate public service announcement messages, satisfied all five selection principles and thus were included in the study. The ethical and socially responsible causes that the focal campaigns advocated were financial literacy and emergency preparedness. Numbers of public service announcements that were available within each medium are provided in Table 1. Each of our 152 respondents provided responses to all 24 ads, resulting in a data set that captured 3648 ratings.

Table 1. Number of public service announcements by medium

\begin{tabular}{lccccc}
\hline & \multicolumn{2}{c}{ Traditional Media } & \multicolumn{2}{c}{ New Media } \\
\hline & $\begin{array}{c}\text { Number of TV } \\
\text { Commercials }\end{array}$ & $\begin{array}{c}\text { Number of Print } \\
\text { Advertisements }\end{array}$ & $\begin{array}{c}\text { Number of Web } \\
\text { Banners }\end{array}$ & $\begin{array}{c}\text { Number of } \\
\text { Online Videos }\end{array}$ & Total \\
\hline Financial Literacy & 3 & 2 & 4 & $4^{\text {a }}$ & 13 \\
\hline Emergency Preparedness & 3 & 2 & 2 & 4 & 11 \\
\hline Total & 6 & 4 & 6 & 8 & 24 \\
\hline
\end{tabular}

\subsection{Procedures}

Each respondent attended a single session in which s/he viewed and provided ratings for all 24 public service announcement messages. Sessions lasted approximately 60 minutes, and participant groups varied in size from 3 to 15 people. Responses were provided in booklets, which included three parts. The 
first section described what to expect in the study, noting specifically that participants would view a number of public service announcements and then record their reactions. A second portion of the response booklet captured attitudes toward the central theme of each public service announcement. Demographic information on age and gender was recorded in the third portion.

Respondents were sequentially shown the public service announcements in orders that were determined by random number assignments at the beginning of each session. Television ads were displayed on a large central monitor. Print ads were projected by a document camera onto a screen. Web banners and online videos were projected from a laptop computer onto this same screen.

After each public service announcement was presented, participants answered three attitudinal questions, utilizing 1-11 semantic differential response scales in which higher scores indicated more favorable attitudes: "My response to this message is unfavorable/favorable," "My feelings toward this message are negative/positive," and "With respect this message, I strongly disagree/agree.

\subsection{Independent Variable}

Conceptually, the central independent variable was message conciseness. This factor was indexed via codings of public service announcement lengths. Two assistants, blind to the purposes of the study, provided length calculations for each message, according to the following instructions. For television commercials and online videos, the unit of measure was number of seconds' duration; for print advertisements and web banners, length was assessed via the number of words appearing in the public service announcement, exclusive of logo nomenclature (e.g., the words from the "Department of Homeland Security" logo were not counted for the emergency preparedness messages). Inter-rater reliability was high, as evidenced by an initial agreement on $92 \%(22 / 24)$ of items coded. Disagreements were resolved via discussion. Per the final message codings, the length ranges represented in the study are listed in Table 2.

Table 2. Length ranges for study messages

\begin{tabular}{cccc}
\hline \multicolumn{2}{c}{ Traditional Media } & \multicolumn{2}{c}{ New Media } \\
\hline $\begin{array}{c}\text { Length of TV Commercials } \\
\text { (seconds) }\end{array}$ & $\begin{array}{c}\text { Length of Print Ads } \\
\text { (words) }\end{array}$ & $\begin{array}{c}\text { Length of Web Banners } \\
\text { (words) }\end{array}$ & $\begin{array}{c}\text { Length of Online Videos } \\
\text { (seconds) }\end{array}$ \\
\hline $10-30$ & $40-55$ & $5-12$ & $180-627$ \\
\hline
\end{tabular}

\subsection{Dependent Variables}

The core dependent variables were attitudes toward the position advocated in each public service announcement. Attitudes have been employed in prior research as an indicator of public service announcement efficacy (e.g., Martiniuk et al. 2010).

Dependent measures were validated by performing a confirmatory factor analysis and calculating Cronbach's $\alpha$ for the battery of three attitude items linked with each public service announcement. Within every set, the three items cohered on a single construct, with loadings above .50, indicating convergent validity (Fornell and Larcker 1981). Moreover, all Cronbach's as were above .70, suggesting appropriate reliabilities (Nunnally and Bernstein 1994). Composite scores were thus computed by internally averaging the attitude ratings.

\subsection{Analysis and Results}

The respondent sample was $56 \%$ male and $44 \%$ female. The median age was 31 . Because multiple attitudinal responses were provided by each participant, repeated-measures analyses of variance were employed to examine data within each medium (web banner, online video, television, print). Detailed results, organized by broad media type (new, traditional) are provided in the sections that immediately follow.

\subsubsection{New Media}


For web banners, omnibus statistical tests confirmed that means differed by message length $(F(5,147)$ $\left.=5.69, p<.01, \eta_{\mathrm{p}}{ }^{2}=.01\right)$. As Figure 1 depicts, attitudes were inversely related to the number of words employed in the public service announcement. That is, shorter web banners were associated with more strongly favorable attitudes. This finding is consistent with Hypothesis 1.

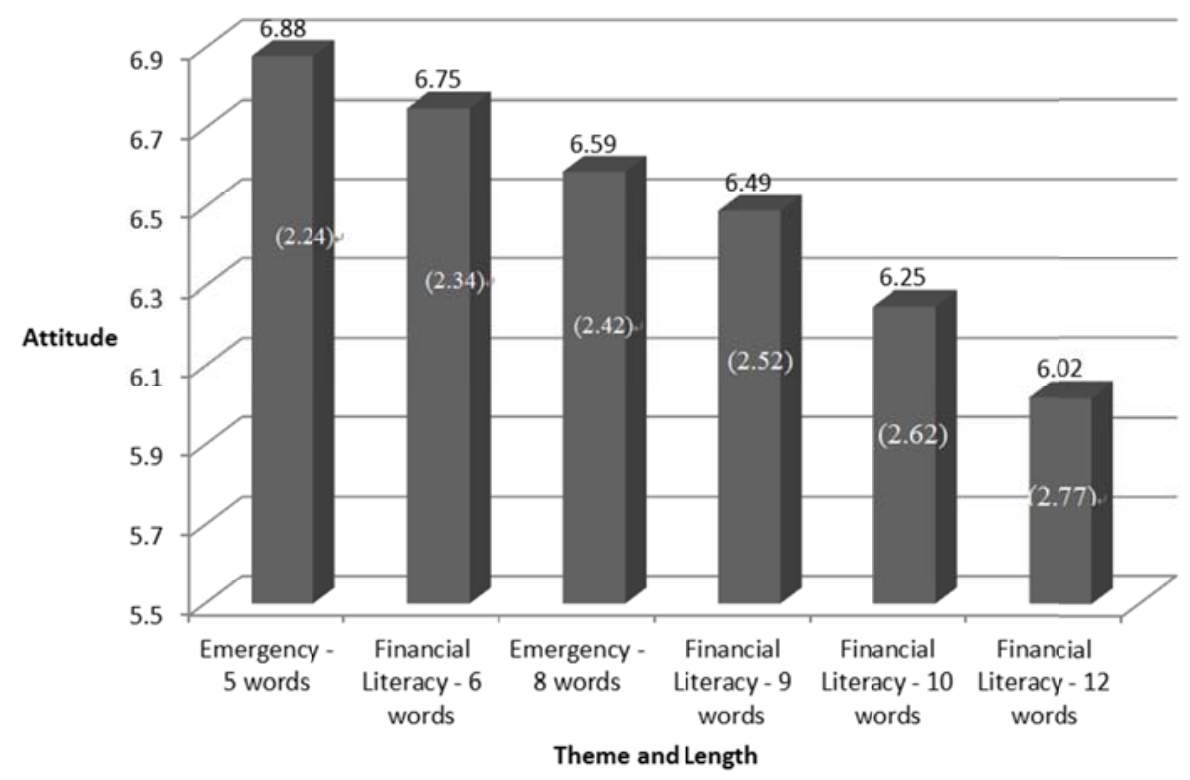

Figure 1. Attitudes by message length: Web banners. Means are labeled above bars. Standard deviations are provided in parentheses.

Planned contrasts were conducted to more intricately explore the differences in means. Results indicate that the downward stair-step pattern of means, arranged from shortest to longest web banner length as in Figure 1, was composed of significant differences in attitudes between each level of word length. That is, the attitudes expressed in response to the 5 -word banner were significantly more favorable than for the 6 -word banner $\left(F(1,151)=5.14, p<.03, \eta_{\mathrm{p}}{ }^{2}=.03\right)$, which was more favorable than for the 8-word banner $\left(F(1,151)=10.84, p<.01, \eta_{\mathrm{p}}{ }^{2}=.07\right)$, which was more favorable than for the 9 -word banner $\left(F(1,151)=16.24, p<.01, \eta_{\mathrm{p}}^{2}=.10\right)$, which was more favorable than for the 10word banner $\left(F(1,151)=18.49, p<.01, \eta_{\mathrm{p}}^{2}=.11\right)$, which in turn, was more favorable than for the 12 word banner $\left(F(1,151)=19.92, p<.01, \eta_{\mathrm{p}}{ }^{2}=.12\right)$. Effect sizes were modest, as is often the case in social scientific research (Rosnow and Rosenthal 2003).

Similarly, for online videos, omnibus tests suggest attitudinal differences by message length, though effect size was again modest (Wilks' $\lambda=.82, F(7,145)=4.47, p<.01 ; \eta_{\mathrm{p}}{ }^{2}=.18$ ). As Figure 2 illustrates, as expected, attitudes were negatively related to public service announcement length. Planned contrasts reveal that the four shortest-length videos (ranging from 180-300 seconds) all produced attitudes that were statistically equal in favorability $(p \mathrm{~s}>.13)$ and that these attitudes were significantly more favorable than those expressed after viewing the longer-length videos (487-627 seconds). Moving from left to right in the Figure 2 graph, each video length beyond 300 seconds produced a statistically significant decrement in attitudes $(p \mathrm{~s}<.03)$. These findings provide additional support for Hypothesis 1. 


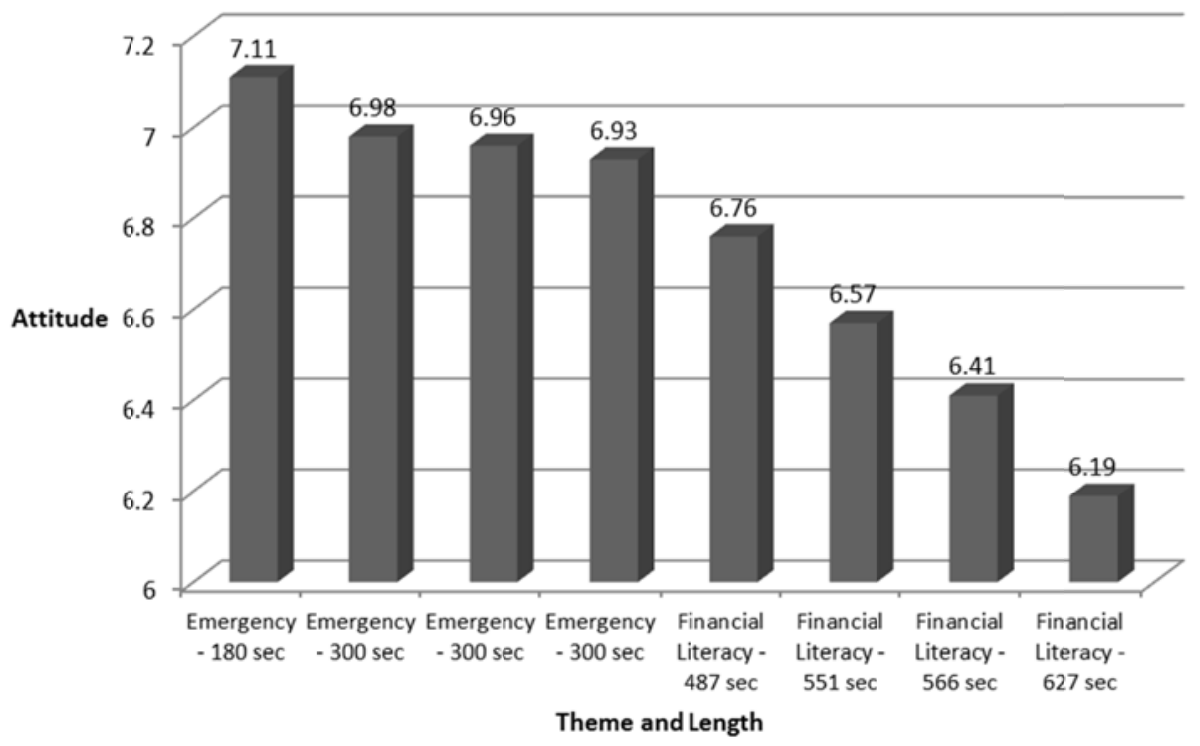

Figure 2. Attitudes by message length: online videos. Means are labeled above bars. Standard deviations are provided in parentheses.

\subsubsection{Traditional Media}

For public service announcements presented as television commercials, omnibus tests again suggest a significant difference in the means for public service announcements with different lengths (Wilks' $\lambda=.18, F(5,147)=133.98, p<.01)$. However, as Figure 3 demonstrates, the pattern was quite opposite to that which was observed for the new media. As predicted by Hypothesis 2, persuasiveness was positively associated with message length. Planned contrasts indicate that attitudes recorded in response to the four 30-second television advertisements did not differ significantly from one another $(p \mathrm{~s}>.25)$ and they were significantly more positive than the attitudes expressed after viewing the 15second TV message $(F(1,151)=301.20, p<.01)$, which in turn, produced significantly more favorable attitudes than the 10 -second TV commercial $(F(1,151)=219.84, p<.01)$.

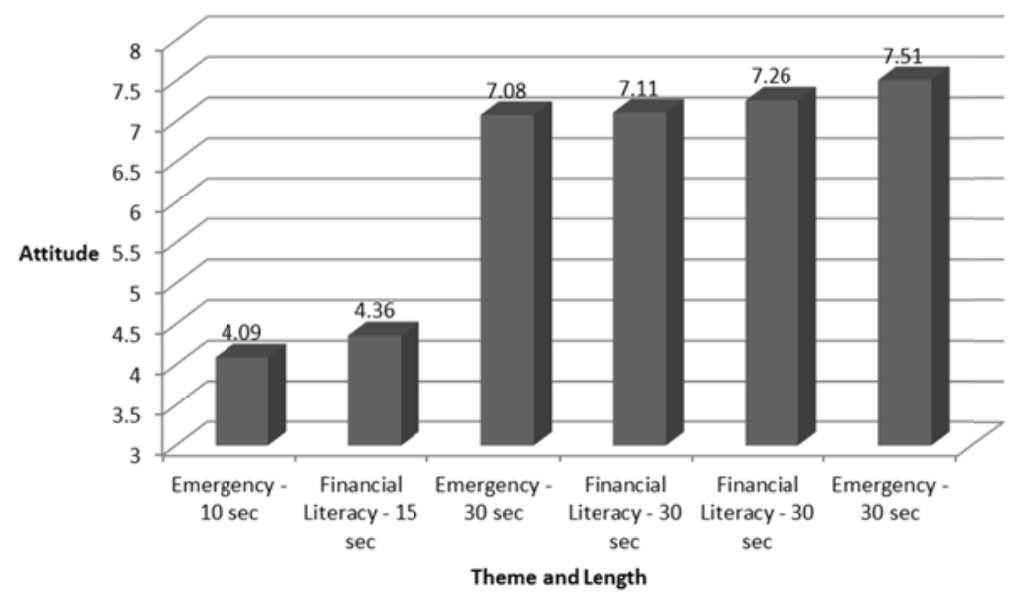

Figure 3. Attitudes by message length: television.

Finally, for the second traditional medium, print, results were conceptually analogous to those observed for television. Omnibus statistics indicate that attitudes differed by message length (Wilks' $\lambda=.19 F(5,14)=126.45, p<.01)$, and inspection of Figure 4 demonstrates that, as anticipated by Hypothesis 2, more positive reactions corresponded to print messages with greater numbers of words. Planned follow-up contrasts more specifically indicate that the three lengthier messages (53-55 words) 
inspired equally favorable attitudes $(p \mathrm{~s}>.24)$ and that these attitudes were significantly stronger than those associated with the shorter print message made up of just 40 words $(F(1,151)=300.66, p<.01)$.

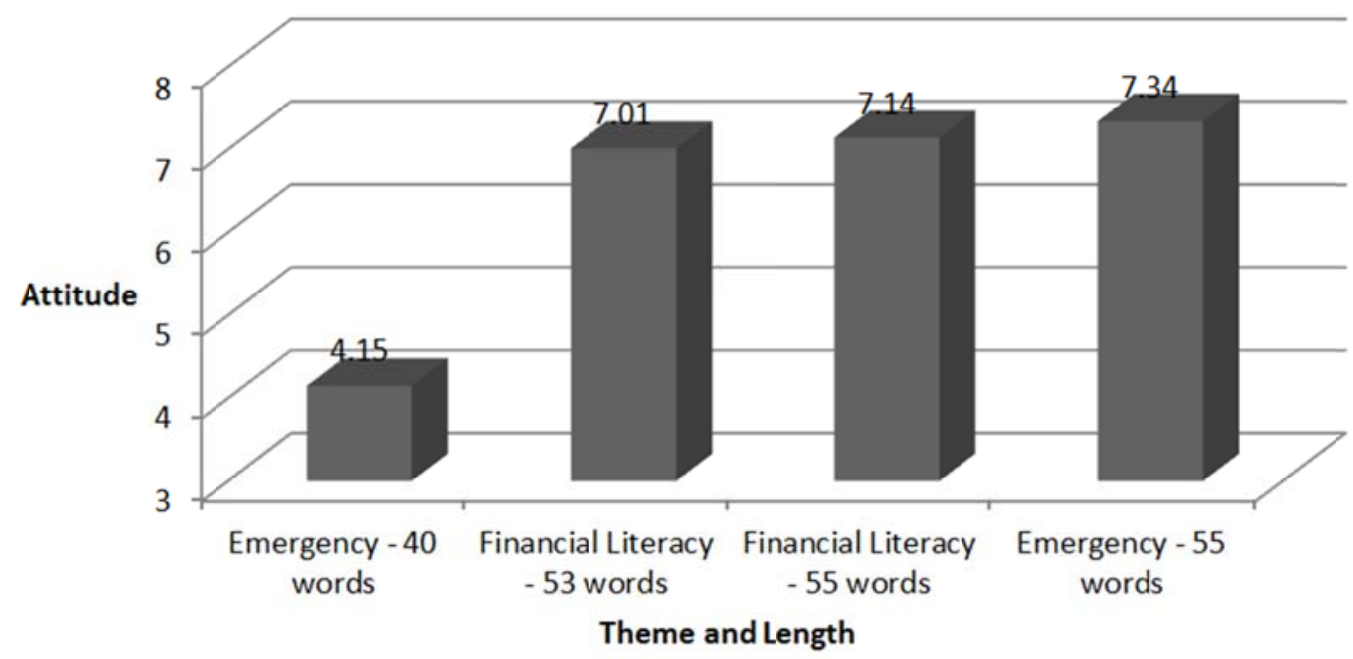

Figure 4. Attitudes by message length: print.

\section{Discussion}

The efficacy of public service announcements has remained a perennial issue of debate (Fishbein et al. 2002; Murry et al. 1996). Given the significant corporate resources that are poured into public service announcement efforts each year, as well as the critical social nature of the problems that are addressed, there is clearly a need for further inquiry into their effectiveness.

The current research has risen to this challenge, focusing particularly on an aspect of public service announcements for which our collective understanding has been, to date, rather constrained. This is, specifically, the dynamics of response to public service announcements that are placed in new media. To gain greater insight into this topic area, we have examined reactions to public service announcements that were delivered through new and traditional media vehicles. Results garnered from testing two forms of new media and two forms of traditional media suggest that persuasiveness varies with message length in opposing directions. For new media, messages that were briefer created a stronger impression, whereas for messages in traditional media, greater duration corresponded to greater impact.

These findings have been explained via a prominent theory of communication norms (Grice 1975), which states that conciseness is among the critical dimensions that people assess in gauging message credibility. Adapting this framework to the new media setting, we have argued that expectations for message length are more truncated in new media, owing to practices that are imposed by prominent players in the domain, such as Twitter. By contrast a "more is better" model of information value was observed for public service announcements placed in traditional media, for which marked expectations of succinctness are considered less likely to govern.

For organizations sponsoring public service campaigns that support ethical and socially responsible causes, the present research may provide useful insights into message development. Whereas a first impulse might be to inundate consumers with reams of information in support of an advocated position, especially when the issue has strong social and ethical implications, this strategy appears to stand at odds with the consumer mentality regarding new media. One observer of the mindset cultivated by platforms such as Twitter described it aptly:

"...the discipline of compression is part of Twitter's charm. Brevity and the management of candour (sic) are essential. One must, as Mark Twain advised, 'eschew surplusage.' Characters count down from 140 above the message window as you type. I imagined that each character costs a dime, and every time I brought my tweet down in size with a disciplined elision, I was saving money..." (Brown 2009). 
In such online communication contexts, brevity is prized, and communications that are short and more pointed are more apt to produce positive responses. This observation begs the practical follow-up question of how a detailed public service announcement might therefore be effectively conveyed to consumer, given the constraints of the new media environment. After all, the nuances of a rich topic such as, say, emergency preparedness may often require fairly intricate transmission of information. For guidance on this issue, we may draw upon other recent findings on online communication, which suggest that consumers may be willing to engage in serial dialogues that become rather extensive over time, even though individual communiqués may be relatively brief (Adjei, Noble and Noble 2010). The picture that emerges from these complimentary research programs is thus that while individual missives benefit from brevity, a full range of intended message points may be delivered through a series of dispatches, each of which may be individually short in duration. A logical trajectory for future inquiry would thus be to explore the utility of social media postings and cause-related blogs, which may offer natural means of engaging audience members in protracted "bite sized" communications on public service topics.

\subsection{Limitations}

Although compelling in their uniformity, our findings are certainly not without their limitations. For example, while the current study has incorporated a range of idiosyncratic executional messages and multiple representations of both traditional and new media, myriad other examples on both accounts await future testing. In supplementary work, a number of intriguing questions might be raised. Will public service announcements for other types of issues conform to the present findings? For instance, might messages related to diseases and other health concerns invoke greater self-interest than the financial literacy and emergency preparedness issues that have been examined herein? Moreover, if that is the case, might tolerance for lengthier messages increase accordingly? In a similar vein, in an increasingly global society, and given prior links between cultural background and processing of web site information (Luna, Peracchio and de Juan 2002), might tolerance for lengthier messages in new media vary according to cultural heritage and associated perspectives on the meaning and role of time? Additional research is warmly encouraged to investigate these and other relevant matters that build upon the current results.

\section{References}

1. Adjei, M. T., Noble, S. M. \& Noble, C. H. (2011). The influence of C2C communications in online brand communities on customer purchase behavior. Journal of the Academy of Marketing Science, 38, 634-653.

2. Anand Keller, P. \& Block, L. G. (1999). Vividness effects: A resource-matching perspective. Journal of Consumer Research, 24 (Dec), 295-304.

3. Brown, I. (2009). Give me twitter or give me death: Six million North Americans now tweet. Is it a bid to stave off the randomness of fate, Ian Brown asks, or is it the tower of babble? The Globe and Mail (March 28), F1.

4. Cacioppo, J. T. \& Petty, R. E. (1982). The need for cognition. Journal of Personality and Social Psychology, 42 (1), 116-131.

5. Chaiken, S. \& Eagly, A. H. (1983). Communication modality as a determinant of persuasion: The role of communicator salience. Journal of Personality and Social Psychology, 45 (2), 241-256.

6. Chaiken, S., Liberman, A. \& Eagly, A. H. (1989). Heuristic and systematic processing within and beyond the persuasion context. In J.S. Uleman \& J.A. Bargh (Eds.) Unintended thought (pp. 212-252), New York: Guilford Press.

7. Eagly, A. H. \& Chaiken, S. (1993). The psychology of attitudes, Fort Worth, TX: Harcourt Brace Jovanovich College Publishers.

8. Fishbein, M., Hall-Jamieson, K., Zimmer, E., von Haeften, I. \& Nabi, R. (2002). Avoiding the boomerang: Testing the relative effectiveness of anti-drug public service announcements before a national campaign. American Journal of Public Health, 92 (2), 238-245.

9. Fornell, C. \& Larcker, D. F. (1981). Evaluating structural equation models with unobservable variables and measurement error. Journal of Marketing Research, 18, 39-50. 
10.Grice, H. P. (1975). Logic and conversation. In P. Cole and J. J. Morgan (Eds.), Syntax and semantics 3: Speech acts (pp. 41-53), New York: Academic Press.

11.Hayes, M. (2009). They will kill us all. The International Herald Tribune, November 13, 8.

12.Jones, G. M. \& Schieffelin, B. B. (2009). Talking text and talking back: "My BFF Jill" from boob tube to YouTube. Journal of Computer-Mediated Communication, 14, 1050-1079.

13.Levine, T. R. \& Hullett, C. R. (2002). Eta squared, partial eta squared, and misreporting of effect size in communication research. Human Communication Research, 28 (4), 612-625.

14.Lorch, E. P., Palmgreen, P., Donohew, L., Helm, D., Baer, S. A. \& Dsilva, M. I. (1994). Program context, sensation seeking, and attention to televised anti-drug public service announcements. Human Communication Research, 20 (3), 390-412.

15.Luna, D., Peracchio, L. A. \& de Juan, M. D. (2002). Crosscultural and cognitive aspects of web site navigation. Journal of the Academy of Marketing Science, 30 (4), 397-410.

16.Martiniuk, A. L. C., Secco, M., Yake, L. \& Speechley, K. N. (2010). Evaluating the effect of a television public service announcement about epilepsy. Health Education Research, 25 (6), 1050-1060.

17.Morgan, S. E., Palmgreen, P., Stephenson, M. T., Hoyle, R. H. \& Lorch, E. P. (2003). Associations between message features and subjective evaluations of the sensation value of antidrug public service announcements. Journal of Communication, 53 (3), 512-526.

18.Murry, J. P., Stam, A. \& Lastovicka, J. L. (1996). Paid- versus donated-media strategies for public service announcement campaigns. Public Opinion Quarterly, 60, 1-29.

19.Nunnally, J. C. \& Bernstein, I. H. (1994). Psychometric theory (pp. 265), New York: McGraw-Hill.

20.O'Dea, J.A. (2005). Prevention of child obesity: First, do no harm. Health Education Research, 20 (2), 259-265.

21.Pechmann, C. \& Knight, S. J. (2002). An experimental investigation of the joint effects of advertising and peers on adolescents' beliefs and intentions about cigarette consumption. Journal of Consumer Research, 29 (June), 519 .

22.Pechmann, C. \& Reibling, E. T. (2006). Antismoking advertisements for youths: An independent evaluation of health, counter-industry and industry approaches. American Journal of Public Health, 96 (5), 906-913.

23.Petty, R. E. \& Cacioppo, J. T. (1986a). Communication and persuasion: Central and peripheral routes to attitude change, New York: Springer-Verlag.

24.Petty, R. E. \& Cacioppo, J. T. (1986b). The elaboration likelihood model of persuasion. In L. Berkowitz (Ed.), Advances in experimental social psychology, 19, (pp. 123-205), San Diego, CA: Academic Press.

25.Petty, R. E., Cacioppo, J.T. \& Schumann, D. W. (1983). Central and peripheral routes to advertising effectiveness: The moderating role of involvement. Journal of Consumer Research, 10 (2), 135-146.

26.Roberto, A. J., Meyer, G., Johnson, A. J., Atkin, C. K. \& Smith, P. K. (2002). Promoting gun trigger-lock use: Insights and implications from a radio-based health communication intervention. Journal of Applied Communication Research, 30 (3), 210-230.

27.Rosnow, R. \& Rosenthal, R. (2003). Effect sizes for experimenting psychologists. Canadian Journal of Experimental Psychology, 57, 221-237.

28.Shanahan, K. J., Hopkins, C. D. \& Carlson, L. (2010). The unintended consequences of using "Posers" in nonprofit public service announcements and proposed self-regulatory disclosure solutions. Journal of Public Policy and Marketing, 29 (2), 219-231.

29.Siu, W. (2010). Interactive source and message effects in persuasion. Perceptual and Motor Skills, 110 (2), 493500 .

30.Toncar, M., Reid, J. S. \& Anderson, C. E. (2007). Effective spokespersons in a public service announcement: National celebrities, local celebrities and victims. Journal of Communication Management, 11 (3), 258-275.

31.Walther, J. B., DeAndrea, D., Kim, J. \& Anthony, J. C. (2010). The influence of online comments on perceptions of antimarajuana public service announcements on YouTube. Human Communication Research, 36, 469-492.

32.Zhao, G. \& Pechmann, C. (2007). The impact of regulatory focus on adolescents' response to antismoking advertising campaigns. Journal of Marketing Research, 44 (Nov), 671-687. 